\title{
Macular Hole in Posterior Microphthalmos
}

\section{Shah $\mathbf{R}^{1 *}$ and Byanju $\mathbf{R}^{2}$}

${ }^{1}$ Department of Ophthalmology, Bharatpur eye hospital, Bharatpur, Chitwan, Nepal

${ }^{2}$ Department of Retina, Bharatpur eye hospital, Bharatpur, Chitwan, Nepal

\begin{abstract}
Posterior microphthalmos is a rare ocular disorder characterized by disproportionately short vitreous length and numerous posterior segment findings. We report a rare coexistence of bilateral macular hole with posterior microphthalmos in a 15-year-old girl. Detailed ophthalmic examination and investigations including B scan ultrasonography and optical coherence tomography were performed. Ophthalmic examination showed bilateral high hyperopia. Best corrected visual acuity was $6 / 18$ in each eye with correction of $+12 D$ in right eye and $+10 D$ in left eye. Ocular biometry measurements showed short total axial length (16.3 $\mathrm{mm}$ both eyes) with remarkable shortening of posterior segment $(9.3 \mathrm{~mm}$ right eye; $9.28 \mathrm{~mm}$ left eye) but normal anterior segment parameters. Anterior chamber depth and horizontal corneal diameter were within normal range. Fundus evaluation revealed bilateral macular hole. Optical coherence tomography finding was compatible with bilateral lamellar macular hole in both eyes. B scan ultrasonography showed sclerochoroidal thickening with foreshortening of vitreous cavity. A variety of posterior segment manifestations associated with posterior microphthalmos have been reported but macular hole is an extremely rare association. This finding is noteworthy and probably among very few in literature.
\end{abstract}

Keywords: Posterior microphthalmos; Macular hole; Optical coherence tomography

\section{Introduction}

Posterior microphthalmos is a rare variety of microphthalmos where the affected eye has a shortening of axial vitreous length thereby resulting in reduction of total axial length but the anterior chamber depth, anteroposterior thickness of the lens and corneal diameter are normal. Prominent features associated with this condition are high hypermetropia and elevated papillomacular retinal fold. Wide variety of posterior segment pathologies associated with this disorder has been reported. We report a very rare coexistence of bilateral macular hole in posterior microphthalmos.

\section{Case presentation}

A 15-year-old girl came for regular eye check-up. She had been using glasses for past 2 years. There was no history of ocular trauma or any systemic illness. Her unconsanguinous parents and only younger brother were healthy and did not use glasses $[1,2]$.

Best corrected visual acuity was $6 / 18$ in each eye with correction of $+12 \mathrm{D}$ in right eye and $+10 \mathrm{D}$ in left eye. The eyes appeared slightly deep set in the orbit with small vertical palpebral fissure height (Figure 1). Anterior segment examination was unremarkable with intraocular pressure, corneal diameter and keratometry readings within normal range. Normal anterior chamber depth was apparent with slit-lamp biomicroscopy and was quantified with optical biometry (Table 1). Fundus evaluation revealed bilateral macular hole (Figure 2). The optic disc appeared normal without crowding of vessels. Although characteristic elevated papillomacular fold of retina was absent, fine retinal folds could

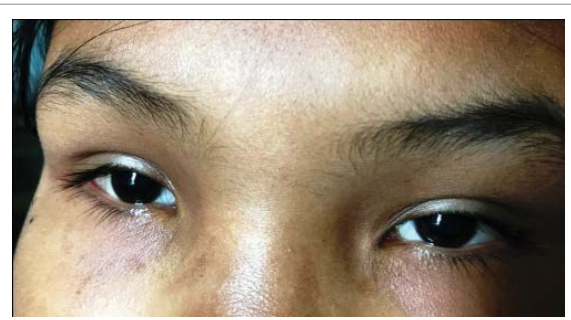

Figure 1: Bilateral deep-set eyes.

\begin{tabular}{|c|c|c|}
\hline Measurement & OD & OS \\
\hline Axial length $(\mathrm{mm})$ & 16.30 & 16.30 \\
\hline Horizontal corneal diameter $(\mathrm{mm})$ & 10.50 & 10.50 \\
\hline AC depth $(\mathrm{mm})$ & 2.96 & 2.94 \\
\hline Lens thickness $(\mathrm{mm})$ & 4.01 & 4.47 \\
\hline Vitreous length $(\mathrm{mm})$ & 9.33 & 9.28 \\
\hline RCS complex thickness $(\mathrm{mm})$ & 2.64 & 2.64 \\
\hline Keratometry & 48.5 at $180^{\circ}$ and & 48.5 at $180^{\circ}$ and 51.5 \\
& 51.5 at $90^{\circ}$ & 14 \\
\hline Intraocular pressure $(\mathrm{mm}$ of $\mathrm{Hg})$ & 13 & 14 \\
\hline
\end{tabular}

Table 1: Biometry measurements.

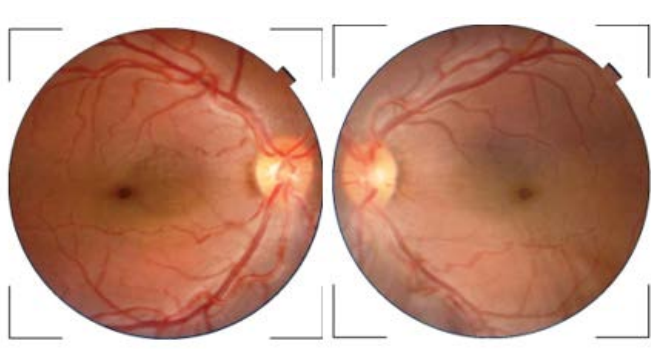

A

B

Figure 2: Fundus photograph shows macular hole with fine retinal folds in both eyes (A) Right eye, (B) Left eye.

be visualized in either eye. There was no evidence of posterior vitreous detachment or peripheral retinal pathology.

Optical coherence tomography finding was compatible with bilateral lamellar macular hole in both eyes (Figure 3). There was no evidence of

*Corresponding author: Shah R, Department of Ophthalmology, Bharatpur eye hospital, Bharatpur-10, Chitwan, Nepal, Tel: 977-9841188085; E-mail: riteshoph@gmail.com

Received July 21, 2018; Accepted July 24, 2018; Published July 30, 2018

Citation: Shah R, Byanju R (2018) Macular Hole in Posterior Microphthalmos. J Clin Case Rep 8: 1147. doi: 10.4172/2165-7920.10001147

Copyright: @2018 Shah R, et al. This is an open-access article distributed under the terms of the Creative Commons Attribution License, which permits unrestricted use, distribution, and reproduction in any medium, provided the original author and source are credited. 


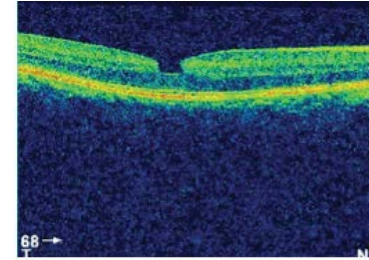

A

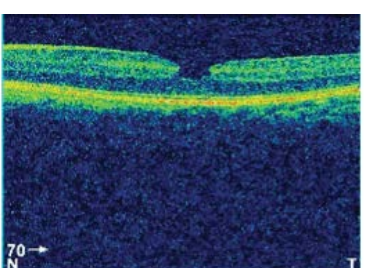

Figure 3: OCT shows bilateral lamellar macular hole (A) Right eye, (B) Left eye.

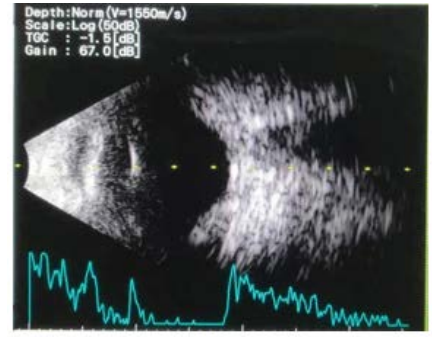

A

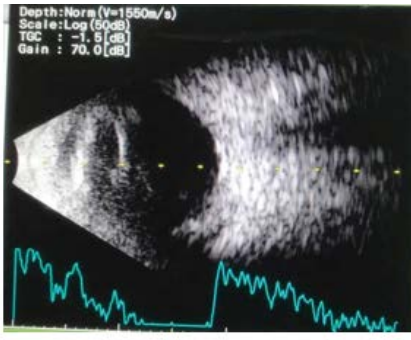

B
Figure 4: B scan ultrasonography shows foreshortening of vitreous cavity and sclerochoroidal thickening in both eyes (A) Right eye, (B) Left eye.

traction or posterior vitreous detachment in either eye. Central foveal thickness was $187 \mu$ in RE and $191 \mu$ in LE. B scan ultrasonography showed sclerochoroidal thickening with foreshortening of vitreous cavity (Figure 4).

The findings were discussed with her parents and in view of her good visual acuity with use of glasses, she was advised for six monthly follow up.

\section{Discussion}

Posterior microphthalmos is a rare developmental ocular disorder characterized by disproportionately shortened posterior segment, high hyperopia and elevated papillomacular retinal fold [3,4]. A diagnosis of posterior microphthalmos was established in this case based on short axial length with marked foreshortening of posterior segment and normal anterior segment dimensions. However, the characteristic elevated papillomacular retinal fold was absent. Nevertheless, fine retinal folds were evident in both eyes. Several cases of posterior microphthalmos with absent papillomacular retinal fold have been reported. Khairallah $\mathrm{M}$ et al. [2] had analysed posterior segment findings in 18 patients with posterior microphthalmos and found that
5 of 18 patients $(27.8 \%$ ) had no elevated papillomacular retinal fold whereas $33.3 \%$ of cases had fine retinal folds. Besides these hallmark findings, numerous other posterior segment associations have been reported by various authors. These include chorioretinal folds, crowded disc, pigmentary retinopathy, absence of capillary free zone $[1,2]$, peripheral retinoschisis, retinal dialysis [5], optic nerve head drusen and foveoschisis [6].

Majority of macular hole are either idiopathic or result from ocular trauma, degenerative myopia and inflammation. Paediatric macular hole is rare and generally associated with trauma [7]. Macular hole in posterior microphtalmos is an extremely rare occurrence. So far only one case of bilateral full thickness macular hole in an eight-yearold Chinese girl with posterior microphthalmos has been reported [8]. In contrast, our case had bilateral lamellar macular hole and the visual acuity was relatively better. The mechanism of macular hole is still unclear. There was no posterior vitreous detachment on indirect ophthalmoscopy and OCT did not reveal any traction in the macular region.

\section{Conclusion}

Although a rare association, macular hole is a noteworthy finding in posterior microphthalmos. Cases with partial thickness hole can have good visual acuity and may only require observation.

\section{References}

1. Spitznas M, Gerke E, Bateman VB (1983) Hereditary posterior microphthalmos with papillomacular fold and high hyperopia. Arch Ophthalmol 101: 413-417.

2. Khairallah M, Messaoud R, Zaouali S, Ben Yahia S, Ladjimi A, et al. (2002) Posterior segment changes associated with posterior microphthalmos. Ophthalmol 109: 569-574.

3. Boynton JR, Purnell EW (1975) Bilateral microphthalmos without microcornea associated with unusual papillomacular retinal folds and high hyperopia. Am J Ophthalmol 79: 820-826.

4. Ryckewaert M, Zanlonghi X, Bertrand-Cuignet H, Constantinides G (1992) High hyperopia with papillomacular fold. Ophthalmologica 204: 49-53.

5. Kim JW, Boes DA, Kinyoun JL (2004) Optical coherence tomography of bilateral posterior microphthalmos with papillomacular fold and novel features of retinoschisis and dialysis. Am J Ophthalmol 138: 480-481.

6. Ayala-Ramirez R, Graue-Wiechers F, Robredo V, Amato-Almanza M, HortaDiez I, et al. (2006) A new autosomal recessive syndrome consisting of posterior microphthalmos, retinitis pigmentosa, foveoschisis, and optic disc drusen is caused by a MFRP gene mutation. Mol Vis 12: 1483-1489.

7. Huang J, Liu X, Wu Z, Sadda S (2010) Comparison of full-thickness traumatic macular holes and idiopathic macular holes by optical coherence tomography. Graefes Arch Clin Exp Ophthalmol 248: 1071-1075.

8. Lee S, Ai E, Lowe M, Wang T (1990) Bilateral macular holes in sporadic posterior microphthalmos. Retina 10: 185-188. 